\title{
Evaluation of anxiety associated with dental visit and treatment experience: A short study
}

\author{
Zehra Shavez, Zareen Fatima* and Afshan Bey
}

Department of Periodontics, Dr Z A Dental College, Aligarh Muslim University, India

\begin{abstract}
Objective: The purpose of this hospital based study was to measure dental anxiety level among patients seeking for dental services.

Methods: The study was based on interviews with 100 patients, aged 20 years-old and over, reporting to Dr Z A Dental College, AMU, Aligarh. Self-administered questionnaire was asked for information about regular dental visits, and dental treatment experience. Beck Anxiety Inventory Scale was applied to measure the anxiety level in the dental patients.
\end{abstract}

Results: It was reported that $66 \%$ of the patients were anxious, according to the Beck Anxiety Inventory Scale. Anxiety was found more in patients who were reporting first time to the dental OPD. Women were more anxious than men at a statistically significant rate and anxiety level was high either at very young or very old age.

Conclusions: Within limits of this study it is concluded that anxiety has significant impact on regular dental visits and dental treatment opted.

\section{Introduction}

Anxiety is an unpleasant state of inner uncertain origin, often accompanied by nervous behaviour. Common symptoms are fear, worry, uneasiness, overreaction to a situation, restlessness, fatigue, problems in concentration. Anxiety in dental clinic may exist due to fear of dental treatment [1].

Dental anxiety has been variously called dental phobia, odontophobia, dentophobia, or dental fear in the literature [2]. Anxiety for dental treatment and the fear of pain remains widespread among patients and is a major inhibiting factor for seeking dental treatment. This study was done to highlight the significance of anxiety associated with regular dental visit and dental treatment experience and thus to focus on the minimizing levels of anxiety and aid in planning stressfree treatment.

\section{Methodology}

A sample of 100 patients was selected aged 20 years and above, reporting to the OPD of Dr Z. A. Dental College, AMU, Aligarh for dental services. Self-administered questionnaire was applied for gaining patient particulars, demographic information, about regular dental visits and dental treatment experience (Short Dental Fear Question, SDFQ) [3]. For measuring the anxiety level in the dental patients Beck Anxiety Inventory Scale was applied. This scale comprised of 21 multiple choice questions related to experience and attitude. Comprehensive statistics was carried out to meet the results.

\section{Results}

The present study reported results from a final sample size of 100 individuals, calculated to measure the level of anxiety in dental patients. Figure 1 shows that the $66 \%$ of the sample population scored high on anxiety scale.
Figure 2 depicts the relation between number of dental visits and anxiety. Anxiety was found more in patients who were reporting first time to the dental OPD. According to Figure 3 women were found more anxious than men. Figure 4 reported the dual peak relationship of anxiety with age. Both very young and very old age patients were found more anxious in comparison to middle age patients.

\section{Discussion}

Anxiety is more associated with first visit because of new

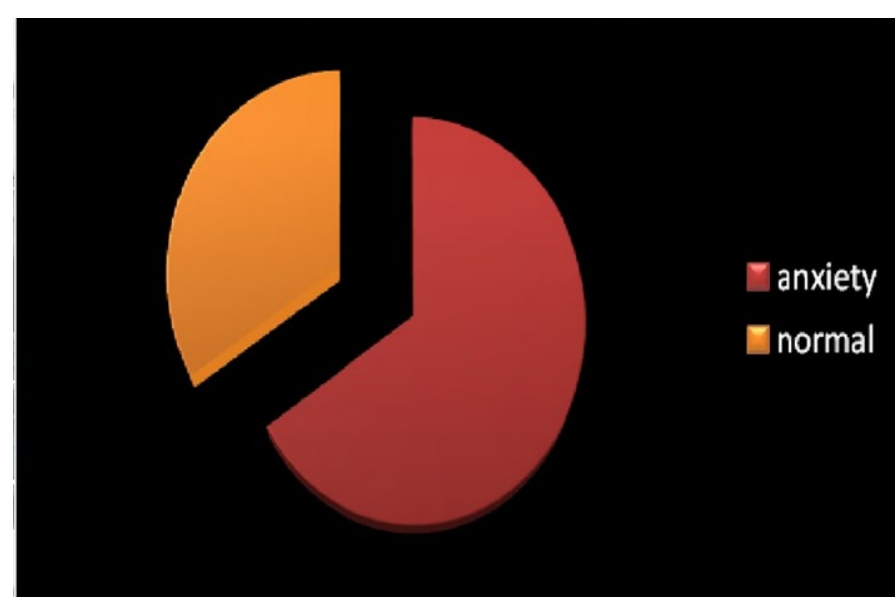

Figure 1. Frequency of anxiety in patients reporting to dental OPD.

Correspondence to: Zareen Fatima, Department of Periodontics, Dr Z A Dental College, Aligarh Muslim University, India, Tel: 09358166068; E-mail: afhanbey@gmail.com

Key words: anxiety, dental visits, fear

Received: May 17, 2015; Accepted: June 20, 2015; Published: June 24, 2015 


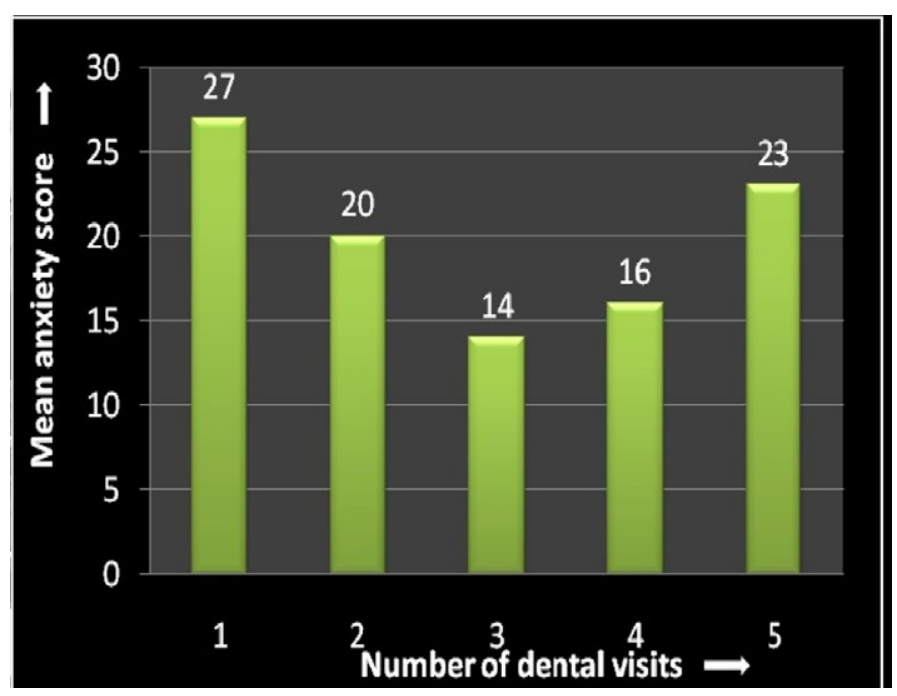

Figure 2. Relationship of anxiety with number of visits.

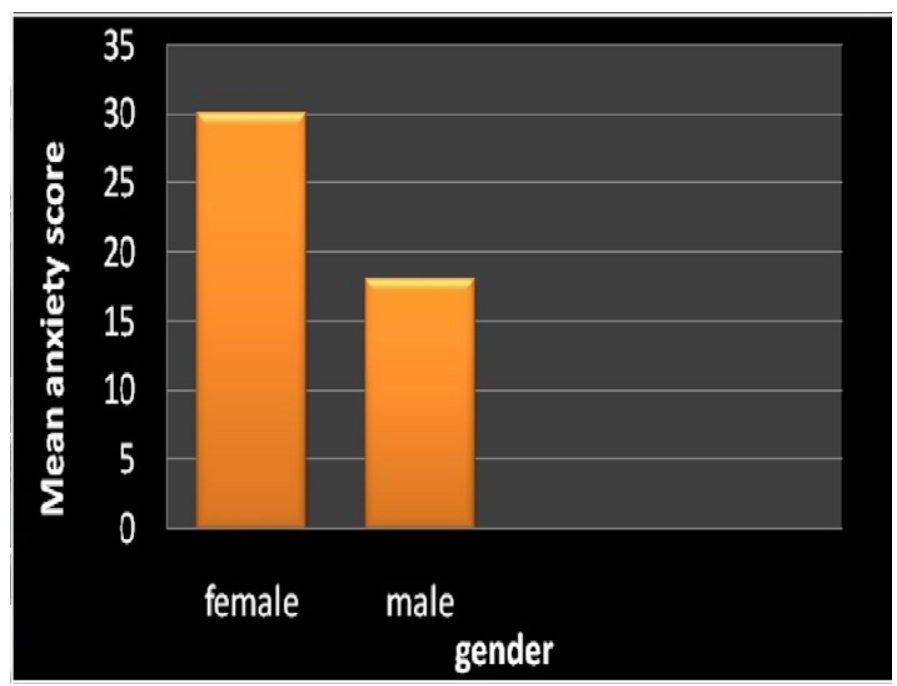

Figure 3. Relationship of anxiety with gender.

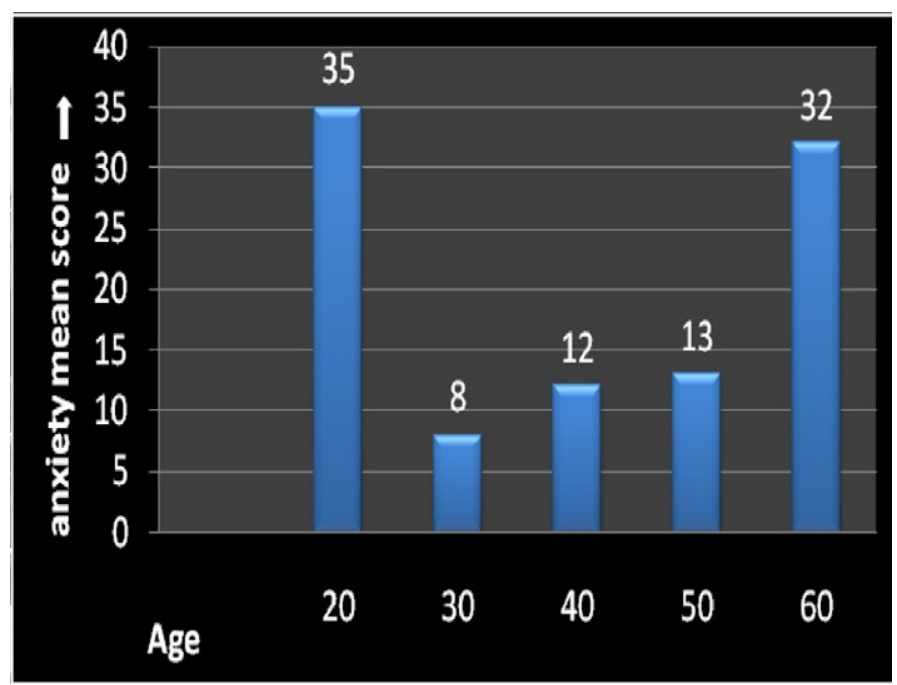

Figure 4. Relationship of anxiety with age. environment and previous heard stories regarding dental treatment [4]. Patient may feel helpless while sitting on a dental chair with wide open mouth, unable to see what's going on inside. Anxiety may also arise due to embarrassment and loss of personal space, physical closeness of the dentist or hygienist to patient's face [5]. On other hand some patients are selfconscious about the appearance of their teeth or possible mouth odours. In last visit of dental treatment there may be chances of anxiety due to final outcome of treatment, its success and treatment need in future.

Female patients are generally more anxious than male patients. It may be due to differences in pain threshold between genders. Women are more sensitive and self-conscious towards their appearance [6]. Previous studies show that older individuals experience lesser anxiety than their younger counterparts due to general decline in anxiety and greater exposure to other diseases and their treatment [7]. In contrast this study shows that very old patients also experience anxiety. This can be explained by differences in attitude towards dental treatment in different demographic regions. Patients over age of 60 generally tend to avoid dental treatment expenses and multiple visits in small towns.

Management for dental anxiety often includes a combination of behavioral and pharmacological methods. Behavioral methods are positive reinforcement (e.g. praising the patient), use of non-threatening language, tell-show-do techniques, relaxation, audio analgesia [8]. An empathic and understanding approach must be taken. Proper time should be given to patient for hearing their queries and normalizing their anxiety. Soft communication and comfortable environment tend to reduce anxiety and speed up the treatment procedure.

Pharmacological methods to manage anxiety in dental clinic are premedication, sedatives and hypnotics, anti histaminic, conscious sedation, general anesthesia [9].

\section{Conclusion}

A significant anxiety may exist at the time of first dental visit or during dental treatment. It is the duty of the dentist to establish a healthy dentist- patient relation and maintain a stress free environment for the patient. Counseling of the patient and maintaining a rapport should an integral part of the dental treatment.

\section{References}

1. Corah NL (1988) Dental anxiety. Assessment, reduction and increasing patient satisfaction. Dent Clin North Am 32: 779-790. [Crossref]

2. Milgrom P, Weinstein $\mathrm{P}$, Getz T. Treating fearful dental patients - a patient management handbook (2nd edn) Seattle: University of Washington.

3. Jaakkola S, Rautava P, Alanen P, Aromaa M, Pienihäkkinen K, et al. (2009) Dental fear: one single clinical question for measurement. Open Dent J 3: 161-166. [Crossref]

4. Locker D, Shapiro D, Liddell A (1996) Negative dental experiences and their relationship to dental anxiety. Community Dent Health 13: 86-92. [Crossref]

5. Humphris G, King K (2011) The prevalence of dental anxiety across previous distressing experiences. J Anxiety Disord 25: 232-236. [Crossref]

6. Economou GC (2003) Dental anxiety and personality: investigating the relationship between dental anxiety and self-consciousness. J Dent Educ 67: 970-980. [Crossref]

7. Liddell A, Locker D (1997) Gender and age differences in attitudes to dental pain and dental control. Community Dent Oral Epidemiol 25: 314-318. [Crossref]

8. Newton T, Asimakopoulou K, Daly B, Scambler S, Scott S (2012) The management of dental anxiety: time for a sense of proportion? Brit Dent J 213: 271-274. [Crossref]

9. Armfield JM, Heaton LJ (2013) Management of fear and anxiety in the dental clinic: a review. Aust Dent J 58: 390-407. [Crossref]

Copyright: $@ 2015$ Shavez Z. This is an open-access article distributed under the terms of the Creative Commons Attribution License, which permits unrestricted use, distribution, and reproduction in any medium, provided the original author and source are credited. 